\title{
In the Extraordinary Times of Coronavirus Disease 2019: Clinical Strategies for Performing Spinal Surgery
}

\author{
Tan Wei Loong Barry ${ }^{1}$, Tan Sze Lyn Jasmin Jeanette ${ }^{2}$, Balakrishnan Ashokka ${ }^{2}$, \\ Keith Gerard Lopez ${ }^{1}$, Joseph Thambiah ${ }^{1}$, Naresh Kumar ${ }^{1}$ \\ ${ }^{1}$ Department of Orthopaedic Surgery, National University Health System, Singapore \\ ${ }^{2}$ Department of Anaesthesia, National University Health System, Singapore
}

The coronavirus disease 2019 (COVID-19) pandemic has caused pronounced strain on global healthcare systems, forcing the streamlining of clinical activities and conservation of health resources. There is a pressing need for institutions to present discipline-specific strategies for the management of COVID-19 patients. We present the comprehensive considerations at the National University Hospital, Singapore from the surgeon's and anesthetist's perspectives in the performance of spinal surgery in COVID-19 patients. These are based on national guidelines and overarching principles of protection for the healthcare workers (HCWs) and efficiency in surgical planning. The workflow begins with the emergency department screening that has been adapted to the local epidemiology of COVID-19 in order to identify suspected/confirmed cases. If patient history cannot be obtained, demographic, clinical, and imaging data are used. Designated orthopedic "contaminated teams" are available 24/7 with an activation time of $<30$ minutes for review. In cases where sub-specialty spine surgeons were required, these professionals were inducted into the "contaminated team" and quarantined until cleared to return to work. Indications for emergency spine surgery were determined pre-emptively. Preoperative surgical considerations included the minimization of manpower, limited dissection, reduced operative time, and judicious use of equipment, leading to reduced aerosolization. Anesthesia considerations include preoperative screening for COVID-19-related concerns that influence surgery, operating room process planning and induction, intraoperative, reversal, recovery, and resuscitation considerations. Focused multi-disciplinary preoperative briefing facilitates familiarization. Surgical, anesthetic, and postoperative workflows were designed to reduce the risk of transmission and protect HCWs while effectively performing spinal surgery. The COVID-19 pandemic has necessitated paradigm shifts in healthcare planning, hospital workflows, and operative protocols. The viral burden does not discriminate between surgeons and physicians, and it is crucial that we, as medical professionals, adapt practices to be malleable and fluid to address the ever-changing developments.

Keywords: COVID-19; Spine surgery; Operative surgical procedures; Perioperative procedures; Anesthesia methods; Hospital planning; Personal protective equipment

Received Jun 23, 2020; Revised Jul 22, 2020; Accepted Jul 27, 2020

Corresponding author: Naresh Kumar

Department of Orthopaedic Surgery, University Orthopaedics, Hand \& Reconstructive Microsurgery Cluster, 1E Kent Ridge Road, NUHS Tower Block, Level 11, Singapore 119228

Tel: +65-67725611, Fax: +65-67780720, E-mail: dosksn@nus.edu.sg 


\section{Introduction}

The coronavirus disease 2019 (COVID-19) pandemic has been labeled by leaders of the modern world as "the greatest challenge of our generation [1]," and "the biggest challenge the world has faced since the second world war [2]," As of 21st July 2020, the global count of confirmed cases was $14,727,752$, with 610,292 reported deaths [3]; representing an alarming exponential increase since its emergence in December 2019 in Wuhan, China. The persistent spread of the pandemic has resulted in international disruptions and those within the medical fraternity, has placed pronounced strain on global healthcare systems [4], forced streamlining of clinical activities [5], encouraged the conservation of health resources [6], and in nearcalamitous situations, necessitated the allocation of finite treatment modalities to seemingly infinite patients based on the likelihood of survival [7].

Thus, there is a pressing need for institutions to present discipline-specific strategies to manage COVID-19 patients. The spine surgeon may not be a frontline worker in the battle against COVID-19; however, considering the complexity and prerequisite skillsets required for spinal surgery; there is a distinct possibility he/she will be called to perform spinal surgery on COVID-19 patients. In this situation, the overarching principles of preservation of health resources, patient and healthcare worker (HCW) protection, and efficiency of surgical planning are crucial. We present our institution's comprehensive considerations during the performance of spinal surgery for COVID-19 patients.

\section{Emergency Department Screening for the Identification of Patients Suspected to Have COVID-19 Infection}

The majority of patients with emergency spine conditions first present to the emergency department (EMD), as per the national guidelines for triage of suspected COVID-19 cases [8]. These guidelines are constantly being updated as per the rapid evolution of the local epidemiology of COVID-19. Unique to Singapore's context, a vast majority of our recently diagnosed patients are from foreign worker (FW) dormitories [9]. The suspect case criteria at the time of writing this manuscript were as follows: (1) clinical signs and symptoms suggesting community-acquired pneumonia or community-acquired severe respiratory in- fection with breathlessness; (2) acute respiratory illnesses of any degree of severity (e.g., symptoms of cough, sore throat, runny nose, and anosmia), with or without fever, who, within 14 days before onset of illness had travelled abroad or come in close contact with a COVID-19 infected person or stayed in a FW dormitory.

Emergency spine surgery is frequently performed in the context of polytrauma. If the relevant history cannot be obtained because of factors such as a low Glasgow Coma Scale, several salient considerations are considered to determine if the patient is a suspect case: patient demographics (e.g., FW living in dormitory), clinical findings (e.g., fever, signs of pneumonia), and imaging results (e.g., chest radiograph and computed tomography [CT]-thorax showing infective changes). After initial assessment, the EMD will decide if the patient requires a review by the orthopedic or spine service.

\section{Initial Point of Contact with Orthopedic Surgery Service}

Our department has a designated "contaminated team" that specifically manages suspects/confirmed COVID-19 cases. The personnel of this department are available 24/7, with an activation time of 30 minutes. These teams comprise an orthopedic consultant (who may or may not be a spine surgeon) and a resident who act as the initial points of contact for the EMD. The "contaminated teams" are rostered on a weekly basis and only review COVID-19/ suspected cases, with minimal contact with their colleagues/patients.

These teams perform the initial assessment of COVID-19 cases with spinal pathologies and conduct the necessary investigations, such as $\mathrm{CT}$ and magnetic resonance imaging scans. If a subspecialty spine surgeon review is required, the case is referred to the on-call spine surgeon who is co-opted into the "contaminated team". He/she is obligated to assess and manage the spinal condition till treatment completion in a confirmed case of COVID-19 and in a suspect case till the case is cleared from having COVID-19, in which case the care is transferred to another spine surgeon in a "clean" team.

\section{Indications for Emergency Spine Surgery}

The explosive development of the COVID-19 pandemic triggered us to pre-emptively determine the following as 
indications for emergency spinal surgery, adapting the North American Spine Society guidelines [10]: (1) rapidly progressive or severe neurologic deficit caused by neurologic compression due to any cause (e.g., infection, tumor, fracture, and disc herniation); (2) spinal instability at high risk of causing neurologic injury or intractable pain due to any cause; (3) infections, such as epidural abscess that require surgical decompression; and (4) postoperative deep wound infection.

\section{Inpatient Assessment and Preparation for Potential Spine Surgery}

All patients are admitted to an isolation ward and are initially assessed by a spine surgeon in full personal protective equipment (PPE) (Fig. 1). The same individual performs the subsequent assessments at regular intervals (if required) in order to minimize the infection risk for the HCW and reduce inter-observer variability. Clinical findings, including the American Spinal Injury Association score, subjective pain score, as well as neurological symptoms and signs were recorded. An anesthetist screens the patient based on electronic records for early patient optimization.

\section{Surgical Team Preoperative Considerations}

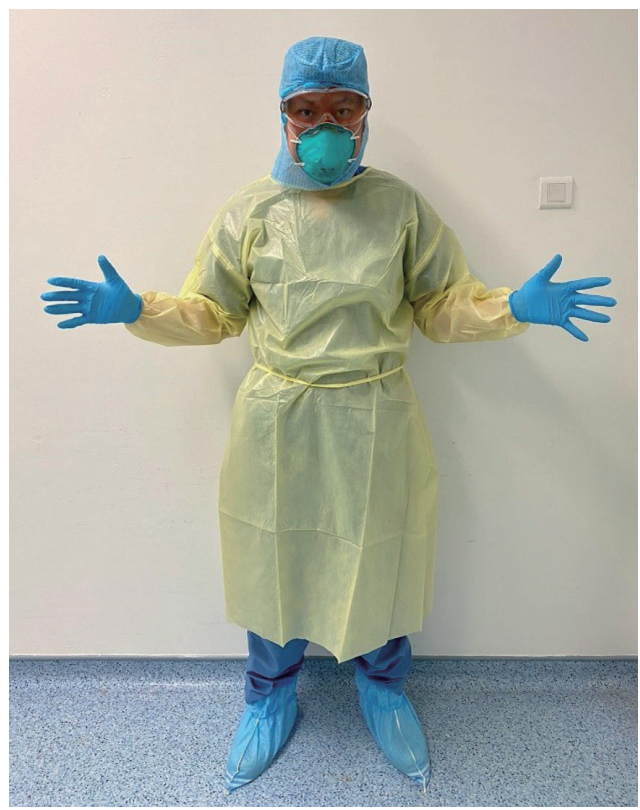

Fig. 1. Personnel in full personal protective equipment.
The surgical plan is dictated by the causative spinal pathology (e.g., unstable fracture and tumor compression), with due consideration for minimizing the extent of surgery, thereby reducing the exposure risks for the HCWs. Operative time and amount of dissection should be minimized. Procedures that lead to aerosolization of microbes raise the chances of infection by 6.6 times [11] and are linked to higher COVID-19 transmission rates $[12,13]$. Attempts are made to minimize the use of equipment that cause aerosolization of microbes. "Damage control surgery [14]," historically used in a polytrauma, describes minimizing of the systemic insult from surgery in an unstable patient; however, in the present context, it can be viewed as the most appropriate surgical plan that aims to achieve a balance between targeted resolution of the spinal pathology and surgical risks to the patient and/ or transmission risks to the HCWs. Minimally invasive surgical techniques can be used but have to be weighed against the generally recognized increase in surgical time and complexity of instruments [15-17].

Once the surgical plan has been finalized, the spine surgeon proceeds to identify the required manpower and equipment. A minimum number of surgical assistants and nurses is recruited into the surgical team. This recruitment will have to be carefully balanced with their experience levels because unfamiliarity will prolong the surgery and increase the risks for the patient and the surgical team [18]. Although not ideal, if circumstances require a prolonged surgery, a standby relieving team may be needed to avoid adverse events that result from fatigue. The pandemic has undoubtedly resulted in an unfamiliar and stressful practicing environment for HCWs, and the physical/mental fatigue should always be considered in surgical planning [19]. Ideally, the team consists of the spine surgeon, the "contaminated team" resident as a single assistant, one scrub nurse, and one circulating nurse.

\section{Special Spinal Equipment for Preoperative Considerations}

\section{Use of surgical microscope}

Decompression usually involves the use of a surgical microscopes viewed through eyepieces. This poses a problem with standard-issue PPE goggles because of the relative distance between the eye and the eyepiece, along with refraction through the lens of the goggles. Advanced micro- 
scope systems (e.g., Kinevo 900; Carl Zeiss Meditec Inc., Jena, Germany) that allow robotic-assisted microscopic magnification with precise, stable and high-definition screen projection (preventing the need for the standard eyepiece) exist and, if available, are a viable option. Alternatives would include surgical loupes that are clipped onto goggles or the performance of decompression after the removal of goggles with rapid replacement after decompression is complete; however, this would result in transient exposure risk.

\section{Use of common surgical tools}

Aerosol-generating equipment, such as electrocautery/ diathermy, should be used in combination with devices that allow local evacuation of surgical smoke, such as a para-incisional smoke evacuator (e.g., miniSQUAIR; Nascent Surgical Inc., Eden Prairie, MN, USA) or a smoke evacuation pencil (e.g., Valleylab; Covidien, Minneapolis, MN, USA). This would theoretically reduce the risk of virus transmission via aerosol transmission [20,21]. Surgeons should limit the use of other aerosol-generating equipment, such as harmonic scalpels, burrs, and saws.

\section{Use of instrumentation systems}

In cases where spinal implants are required, the spine surgeon should select a system that he/she is familiar with and ideally would not require the assistance of an implant vendor. If an implant vendor is required, they must be trained in the use of PPE.

\section{Use of neuromonitoring}

Ideally, the surgery is performed without neuromonitoring unless there are strong indications. Our department uses neuromonitoring only in cases of complex/revision surgery or extensive spinal instrumentation. Neuromonitoring also necessitates the use of "total intravenous anesthesia" that depletes precious supplies of drugs, such as propofol, that are essential in intensive care units (ICUs). The neuromonitoring technician should be trained in the use of PPE and be familiar with the operating room (OR) protocols. After the neuromonitoring requirements are complete, the technician should safely exit the theatre appropriately.

\section{Anesthesia Considerations}

\section{Preoperative screening}

A thorough preoperative evaluation is performed to screen for infection-related concerns that influence the course of the surgery. These include the stratification of the severity of systemic involvement in COVID-19, the presence of cardiovascular manifestations [22], such as hypertension (owing to interactions with angiotensin converting enzyme), viral myocarditis, and hemodynamic compromise from intercurrent co-infections and septicemia. Multisystem presentations vary, based on the degree of host-immune responses. Cytokine storm is implicated in cardiac failure, severe acute respiratory distress syndrome (ARDS), liver and kidney failure, and procoagulant state that results in microthrombi formation that require systemic anticoagulation [23-26]. As spine surgeries require the use of potent intravenous anesthetic agents with anticipated hemodynamic changes, patients need to have adequately optimized end-organ function. Complete blood count, clotting functions, renal function, and cardiac evaluations, such as electrocardiography and echocardiography are performed as required. Preoperative hydration and nutrition are optimized, and surgery is scheduled as per fasting guidelines to avoid pulmonary complications from aspiration.

\section{Operating room process planning}

A negative pressure OR that has a minimum of 6-12 cycles of air change per hour is prepared. While we maximize the use of disposable equipment, non-disposable equipment, such as anesthesia machines need to be protected by plastic films or scaffolds for later re-use. The accompanying personnel should wear complete PPE. Powered air-purifying respirators (PAPRs) are worn by the anesthesia team for intubation and airway manipulations (Fig. 2).

The patient is then brought down to the OR directly, bypassing the usual holding/pre-induction areas via an evacuated pathway to minimize the exposure to HCWs on the way [27]. After the completion of preliminary checks to ascertain patient identity and site of surgery, detailed reviews are conducted within the OR. 


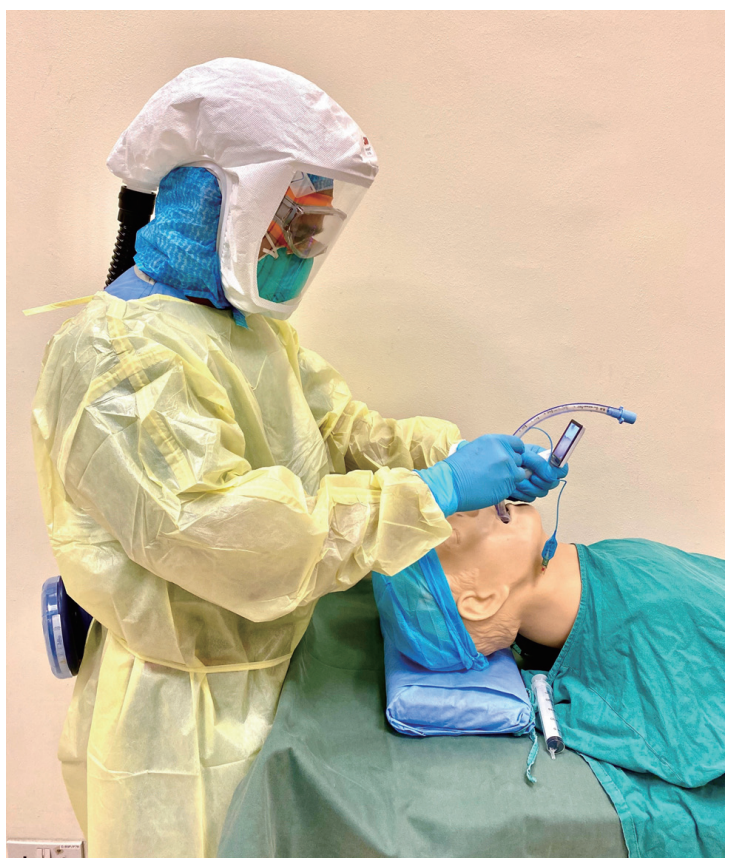

Fig. 2. Intubation procedure by an anesthetist in powered air-purifying respirator.

\section{Induction and intraoperative considerations}

General anesthesia is the standard choice for spinal surgeries. Intraoperative considerations aim to prevent contamination of the anesthetic machine, equipment, and $\mathrm{OR}$, and to minimize exposure to the HCW [28]. The most experienced anesthetist performs the intubation with the routine use of a video laryngoscope to minimize the number of attempts, circumvent potential visual difficulties related to the barrier precautions, and avoid close proximity of the anesthetist's face with the patient's oropharynx. A depiction of the standard intubation procedure has been demonstrated in Fig. 2. Non-anesthesia personnel should exit the room and only enter $20 \mathrm{~min}$ utes after the airway is secured to allow sufficient OR airexchanges.

Preoxygenation with $100 \%$ oxygen is performed for 3-5 minutes; this permits longer time for intubation, and minimizes the likelihood of desaturation that would require rescue mask ventilation. Intubation is performed with "rapid sequence induction" whereby the pre-calculated doses of anesthetic agents are administered without mask ventilation after administering rapid-onset muscle relaxants. Once the endotracheal tube (ETT) is inserted, the cuff is inflated immediately and checked for seal before connecting to the anesthetic circuit or making any venti- latory attempts. Placement is confirmed via end-tidal capnography, observation of bilateral chest rise, and normal ventilation parameters. Auscultation using a stethoscope might involve a risk of contamination of the clinician's face near the ear region and might not be feasible if one is wearing the PAPR or other helmet devices.

Prevention of cross contamination of the anesthetic machine and the vicinity of the patient is achieved with the use of at least two high-efficiency particulate air/heat and moisture exchanger filters as per the recommended guidelines [29]: one filter is placed at the patient end of the breathing circuit after the Y-piece connector, and the second filter is placed on the expiratory limb of the breathing circuit. When neuromonitoring is used, the anesthetic depth is monitored with processed electroencephalograms, such as bispectral index score. This would ensure that patients are adequately anesthetized or sufficiently paralyzed to prevent coughing (aerosol generation), dangerous circuit disconnections, and ETT dislodgement. Depth of paralysis, when used, should be monitored intraoperatively, and muscle relaxants should be administered appropriately. Lung-protective ventilation strategies can be considered in the place of patients' risk of developing ARDS. Disconnection of the circuit should be minimized; if necessary, one should clamp the ETT/circuit or suspend the oxygen flow to prevent aerosolization. This is particularly important because spine surgery often requires the patient to be in the prone position that requires transient disconnection of the circuit.

\section{Reversal considerations}

Only the anesthesia personnel remain in the theatre during reversal. Anesthetic techniques to minimize coughing and vomiting, such as adequate analgesia, full reversal of residual paralysis, and prophylactic administration of antiemetics for smooth emergence, are employed. The use of non-invasive ventilation devices, such as bi-level positive airway pressure/continuous positive airway pressure, require stringent patient selection (for the perfect seal around the face) and should be minimally used unless performed in negative pressure isolation rooms owing to the inherent risk of aerosolization [30].

\section{Recovery considerations}

Recovery of the patient should occur within the same OR 


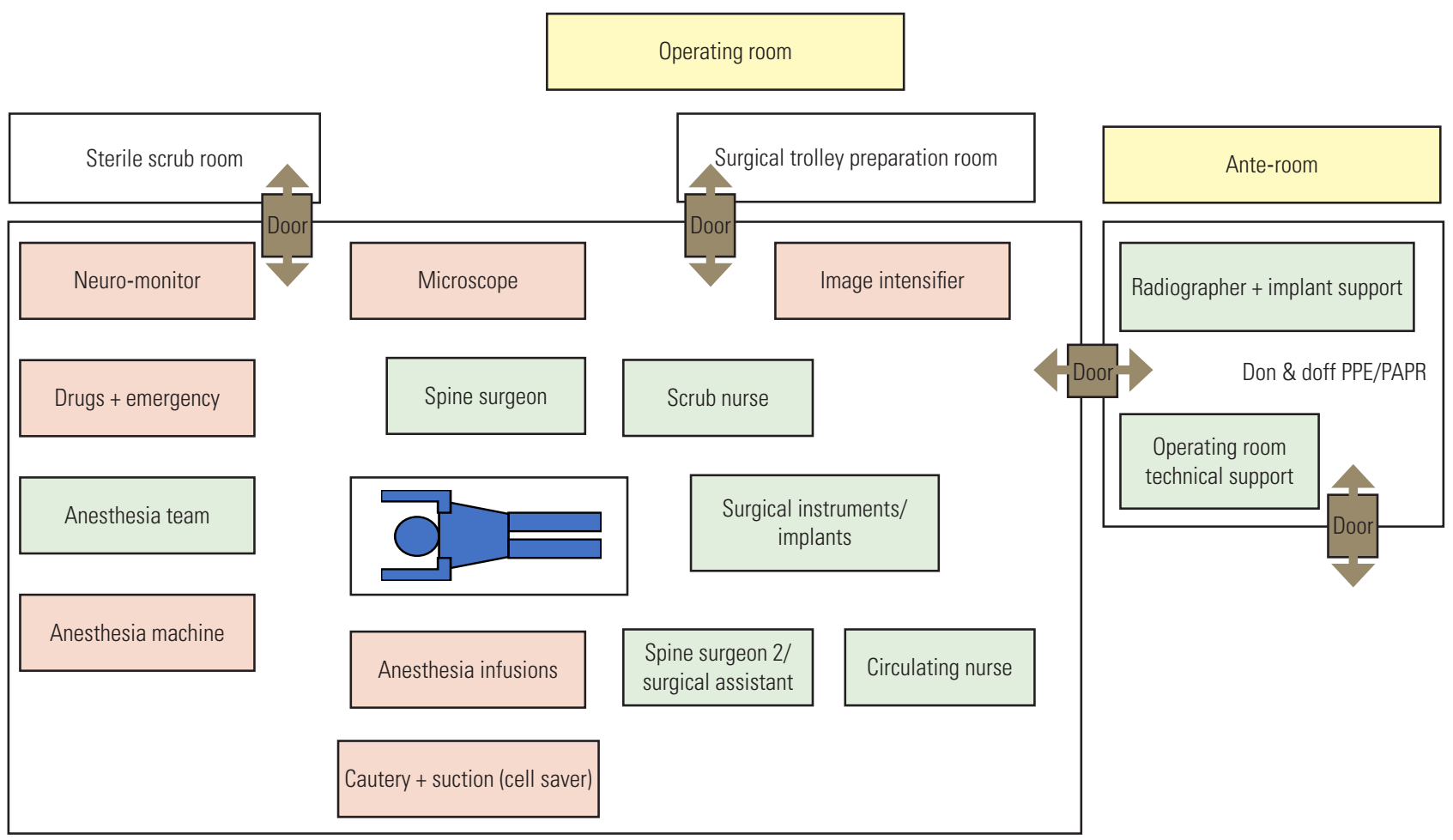

Fig. 3. Standard operating room layout for spine surgery in coronavirus disease 2019 patient. PPE, personal protective equipment; PAPR, powered air-purifying respirator.

with transport of the patient directly back to an isolation ward. High-touch surfaces and reusable equipment should be thoroughly disinfected using 5,000 ppm of sodium dichloroisocyanurate. The floors are to be cleaned with 1,000 ppm of sodium dichloroisocyanurate [31]. The anesthesia breathing circuit, reservoir bag, mask, forced air warming blanket, and other disposable equipment should be discarded. After the patient leaves the OR, the OR needs to be allowed air circulation for 1 hour before the next procedure.

\section{Resuscitation considerations}

If resuscitation is required during the OR process or in the immediate postoperative period, chest compressions without rescue/mouth-to-mouth respirations are performed. Aerosol-generating procedures, such as intubation, should only be attempted after the clinicians have appropriately worn the complete PPE. Mask ventilation should be avoided, and if necessary, a two-person technique is advised. Modified resuscitation guidelines are proposed where early airway access and securing of the trachea with cuffed ETT is performed to prevent aerosolization.

\section{Surgical Preoperative Briefing}

Once the anesthetic and operative plans are complete, the relevant manpower recruited and equipment prepared, and we recommend focused preoperative briefing. The case is succinctly discussed; any doubts are clarified, and potential pitfalls are identified. The spine surgeon would come into contact with the patient; therefore, the current "social distancing measures" permeating work practice, preoperative briefings should be performed via teleconferencing platforms. The workflow and PPE guidelines are also refreshed. It is imperative to involve the relevant multi-disciplinary theatre staff who may not be physically present at the time of the surgery, including the OR nurse managers and high-dependency (HD)/ICU teams.

\section{Operating Room Overview}

A standard COVID-19 OR set up at the National University Hospital, Singapore has been presented in Fig. 3. This set-up is customized as per each patient, and the final layout (modified as per the preoperative briefing) should be shown at the end of the preoperative briefing. 


\section{Spine Surgery Surgical Workflow}

Our institution has a dedicated negative-pressure theatre reserved during this pandemic for COVID-19 patients. This OR is located within the same complex, but at a separate location with specific access corridors. When the patient is being brought to the OR complex, the surgical team and involved OR staff are already dressed in complete PPE and are waiting in the ante room. The complete PPE involves an N95 respirator (National Institute for Occupational Safety and Health-approved respirator), protective goggles, surgical tie-on hood, a disposable longsleeved waterproof gown over a standard short sleeved scrub shirt and trousers, gloves, and shoe covers over surgical boots or shoes (Fig. 1). The OR staff in full PPE then enter the OR and transfer the patient onto the operating table for induction. For prone-position surgery, the patient is induced on the trolley bed before being transferred to the operating table. The surgical team only enters the OR 20 minutes after intubation, as described in the section of anesthesia considerations.

The patient is then positioned by the surgical team that is dressed in complete PPE, and after positioning, the surgical team proceeds to remove their external long-sleeved gown and gloves in the OR, perform appropriate handwashing with an alcohol-based chlorhexidine gluconate solution, and then return to the scrub room for surgical scrubbing and gowning. If a lead gown is required, it is worn before scrubbing. Reinforced surgical gowns, with length up to the ankles are worn, and at least two pairs of gloves are worn to create an internal layer and an external layer (Fig. 4).

During the surgical procedure, electrocautery settings are set at the suitably lowest level, and local smoke evacuation devices are used as available, with liberal use of standard surgical field suction catheters. The OR technician performs scheduled checks of the circuits to ensure appropriate functioning. As mentioned, the use of aerosol generating tools, such as saws, burrs and drills are minimized, and if needed, used at the lowest speed possible.

If an imaging intensifier is required, once complete, the radiographer is asked to remove his PPE appropriately and exit the OR expediently. Microscope use, if necessary, is kept to a minimal duration if PPE goggles are removed during microscope use. The PPE googles are immediately replaced by the circulating nurse once the microscoperequiring procedure is completed.

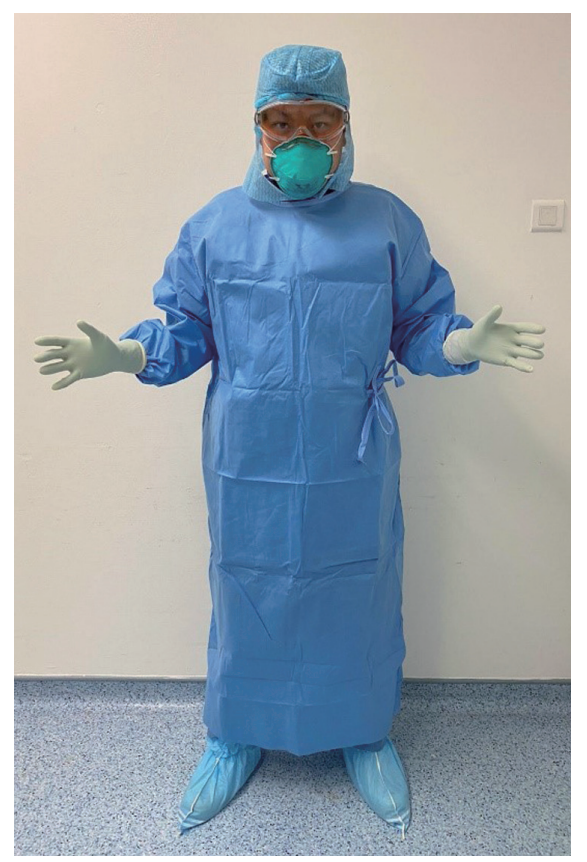

Fig. 4. Surgical team attire (lead gown worn as inner layer).

Before performing wound closure, the surgeon will inform the anesthetist early for postoperative planning. The circulating nurse will also be signaled to inform the external OR reception to arrange the necessary transfer logistics. This chain of communication is necessary to minimize unnecessary waiting times where there is a risk of infection transmission from the patient to the surrounding environment.

\section{Postoperative Workflow}

At the end of the procedure, the surgical team first proceeds to remove their gloves and surgical gown in the OR. An alcohol-based chlorhexidine gluconate solution is used for hand disinfection. The surgical team then steps into an ante room with appropriate waste baskets and removes the lead gown (if worn), surgical hood, shoe covers, goggles, and finally the N95 respirator. At each step of removal, the hands are disinfected. Thereafter, they exit toward the change rooms to shower.

Operative notes are not documented in the computer terminals at the same OR; however, they are remotely documented at a later stage at the OR reception because the anesthetists then reverse the patient in the OR, and the technicians clean the theatre thereafter.

The postoperative review requires an assessment of the power and sensation. This needs to be performed after the 
patient is acceptably conscious and can obey instructions to minimize the need for repeated reviews. The anesthetist will thus inform the surgeon a suitable time for assessment, based on the expected time of anesthesia recovery. A single surgeon in complete PPE assesses the patient after direct transfer to the isolation ward. Where appropriate, measurement of parameters should be performed at reasonable intervals to minimize the risk of exposure to the nursing staff. Alternatively, the patient can be placed in a HD or ICU facility with remote monitoring, especially if invasive lines, such as arterial lines are already placed. Postoperative imaging should also be scheduled only if necessary.

\section{Conclusions}

The COVID-19 pandemic has necessitated paradigm shifts in healthcare planning, hospital workflow, and operative protocols. The viral burden does not discriminate between surgeons and physicians, and it is crucial for medical professionals to adapt our practices to be malleable and fluid and thus address the ever-changing developments. With the ongoing pandemic, this method of trial by fire may present COVID-19 surgical cases that require meticulous planning by spine surgeons as they continue to perform their duties of patient care.

\section{Conflict of Interest}

No potential conflict of interest relevant to this article was reported.

\section{Acknowledgments}

We would like to acknowledge Dr Mark Chong, Dr Zhen Chang Liang, Dr Diarmuid Murphy and Dr James Hui for their contributions in providing details for the workflow in the 'contaminated teams.

\section{References}

1. Londono E, Andreoni M, Casado L. Bolsonaro, isolated and defiant, dismisses coronavirus threat to Brazil. The New York Times [Internet]. 2020 Apr 1 [cited $2020 \mathrm{Jul}$ 22]. Available from: https://www. nytimes.com/2020/04/01/world/americas/brazilbolsonaro-coronavirus.html.
2. The Economic Times. Merkel calls coronavirus 'biggest challenge since WWII'. The Economic Times [Internet]. 2020 Mar 19 [cited 2020 Jul 22]. Available from: https://economictimes.indiatimes.com/news/ international/world-news/merkel-calls-coronavirusbiggest-challenge-since-wwii/articleshow/74702583. cms.

3. Center for Systems Science and Engineering. COVID-19 dashboard by the Center for Systems Science and Engineering (CSSE) at Johns Hopkins University (JHU). Baltimore (MD): Coronavirus Resource Centre, Johns Hopkins University and Medicine; 2020.

4. Emanuel EJ, Persad G, Upshur R, et al. Fair allocation of scarce medical resources in the time of Covid-19. N Engl J Med 2020;382:2049-55.

5. Coccolini F, Perrone G, Chiarugi M, et al. Surgery in COVID-19 patients: operational directives. World J Emerg Surg 2020;15:25.

6. Swiss Academy of Medical Sciences. COVID-19 pandemic: triage for intensive-care treatment under resource scarcity. Swiss Med Wkly 2020;150:w20229.

7. Monella LM. Coronavirus: Italy doctors 'forced to prioritise ICU care for patients with best chance of survival'. Euronews [Internet]. 2020 Mar 13 [cited 2020 Jul 22]. Available from: https://www.euronews. com/2020/03/12/coronavirus-italy-doctors-forcedto-prioritise-icu-care-for-patients-with-best-chanceof-s.

8. Ministry of Health Singapore. MOH circular 97A/ 2020: revision of suspect case definition for coronavirus disease 2019 (COVID-19). Singapore: Ministry of Health Singapore; 2020.

9. Channel News Asia. 1,426 New COVID-19 cases in Singapore, mostly foreign workers in dormitories. Channel News Asia [Internet]. 2020 Apr 20 [cited 2020 Jul 22]. Available from: https://www.channelnewsasia.com/news/singapore/covid-19-newcases-1426-foreign-workers-dormitory-citizen-prmoh-12658250.

10. North American Spine Society. COVID-19: resources and updates. Burr Ridge (IL): North American Spine Society; 2020.

11. Tran K, Cimon K, Severn M, Pessoa-Silva CL, Conly J. Aerosol generating procedures and risk of transmission of acute respiratory infections to healthcare workers: a systematic review. PLoS One 2012;7:e35797. 
12. Tompkins BM, Kerchberger JP. Special article: personal protective equipment for care of pandemic influenza patients: a training workshop for the powered air purifying respirator. Anesth Analg 2010;111:93345.

13. Peng PW, Ho PL, Hota SS. Outbreak of a new coronavirus: what anaesthetists should know. $\mathrm{Br} J$ Anaesth 2020;124:497-501.

14. Giannoudis PV. Surgical priorities in damage control in polytrauma. J Bone Joint Surg Br 2003;85:478-83.

15. Kumar N, Malhotra R, Maharajan K, et al. Metastatic spine tumor surgery: a comparative study of minimally invasive approach using percutaneous pedicle screws fixation versus open approach. Clin Spine Surg 2017;30:E1015-21.

16. Kumar N, Malhotra R, Zaw AS, et al. Evolution in treatment strategy for metastatic spine disease: presently evolving modalities. Eur J Surg Oncol 2017;43:1784-801.

17. Lee LY, Idris Z, Beng TB, et al. Outcomes of minimally invasive surgery compared to open posterior lumbar instrumentation and fusion. Asian J Neurosurg 2017;12:620-37.

18. Finnesgard EJ, Pandian TK, Kendrick ML, Farley DR. Do not break up the surgical team!: familiarity and expertise affect operative time in complex surgery. Am J Surg 2018;215:447-9.

19. Janhofer DE, Lakhiani C, Song DH. Addressing surgeon fatigue: current understanding and strategies for mitigation. Plast Reconstr Surg 2019;144:693e$699 \mathrm{e}$.

20. Georgesen C, Lipner SR. Surgical smoke: risk assessment and mitigation strategies. J Am Acad Dermatol 2018;79:746-55.

21. Ulmer BC. The hazards of surgical smoke. AORN J 2008;87:721-34.

22. Basu-Ray I, Almaddah NK, Adeboye A, Soos MP. Cardiac manifestations of coronavirus (COVID-19). Treasure Island (FL): StatPearls Publishing; 2020.
23. Becker RC. COVID-19 update: COVID-19-associated coagulopathy. J Thromb Thrombolysis 2020;50:5467.

24. Nile SH, Nile A, Qiu J, Li L, Jia X, Kai G. COVID-19: pathogenesis, cytokine storm and therapeutic potential of interferons. Cytokine Growth Factor Rev 2020;53:66-70.

25. Zaim S, Chong JH, Sankaranarayanan V, Harky A. COVID-19 and multiorgan response. Curr Probl Cardiol 2020;45:100618.

26. Zheng YY, Ma YT, Zhang JY, Xie X. COVID-19 and the cardiovascular system. Nat Rev Cardiol 2020;17:259-60.

27. Ti LK, Ang LS, Foong TW, Ng BS. What we do when a COVID-19 patient needs an operation: operating room preparation and guidance. Can J Anaesth 2020;67:756-8.

28. Wax RS, Christian MD. Practical recommendations for critical care and anesthesiology teams caring for novel coronavirus (2019-nCoV) patients. Can J Anaesth 2020;67:568-76.

29. Zucco L, Levy N, Ketchandji D, Aziz M, Ramachandran SK. Perioperative considerations for the 2019 novel coronavirus (COVID-19). Rochester (MN): Anesthesia Patient Safety Foundation; 2020.

30. Ashokka B, Loh MH, Tan CH, et al. Care of the pregnant woman with coronavirus disease 2019 in labor and delivery: anesthesia, emergency cesarean delivery, differential diagnosis in the acutely ill parturient, care of the newborn, and protection of the healthcare personnel. Am J Obstet Gynecol 2020;223:66-74.

31. Ong SW, Tan YK, Chia PY, et al. Air, Surface environmental, and personal protective equipment contamination by severe acute respiratory syndrome coronavirus 2 (SARS-CoV-2) from a symptomatic patient. JAMA 2020;323:1610-2. 